\title{
Stable invariant subspaces for operators on Hilbert space
}

\author{
by John B. Conway (Knoxville, Tenn.) and \\ Don Hadwin (Durham, N.H.)
}

\begin{abstract}
If $T$ is a bounded operator on a separable complex Hilbert space $\mathcal{H}$, an invariant subspace $\mathcal{M}$ for $T$ is stable provided that whenever $\left\{T_{n}\right\}$ is a sequence of operators such that $\left\|T_{n}-T\right\| \rightarrow 0$, there is a sequence of subspaces $\left\{\mathcal{M}_{n}\right\}$, with $\mathcal{M}_{n}$ in Lat $T_{n}$ for all $n$, such that $P_{\mathcal{M}_{n}} \rightarrow P_{\mathcal{M}}$ in the strong operator topology. If the projections converge in norm, $\mathcal{M}$ is called a norm stable invariant subspace. This paper characterizes the stable invariant subspaces of the unilateral shift of finite multiplicity and normal operators. It also shows that in these cases the stable invariant subspaces are the strong closure of the norm stable invariant subspaces.
\end{abstract}

Throughout this paper, $\mathcal{H}$ is a separable complex Hilbert space and $\mathcal{B}(\mathcal{H})$ is the algebra of bounded operators on $\mathcal{H}$. All notation and terminology used here will be that of [6]. In particular, Lat $T$ denotes the lattice of invariant subspaces of $T$ for any operator $T$ in $\mathcal{B}(\mathcal{H})$.

Usually, a closed subspace $\mathcal{M}$ of $\mathcal{H}$ will be identified with the orthogonal projection onto it, $P_{\mathcal{M}}$. So, in particular, the collection of all closed subspaces of $\mathcal{H}$ will be identified with the collection $\mathcal{P}$ of all projections in $\mathcal{B}(\mathcal{H})$. When the convergence of a sequence of subspaces in the norm or strong topology is mentioned, this is actually a statement about the corresponding convergence of the associated sequence of projections.

If $T$ is a bounded operator on $\mathcal{H}$, an invariant subspace $\mathcal{M}$ for $T$ is stable provided whenever $\left\{T_{n}\right\}$ is a sequence of operators such that $\left\|T_{n}-T\right\| \rightarrow 0$, there is a sequence of subspaces $\left\{\mathcal{M}_{n}\right\}$, with $\mathcal{M}_{n}$ in Lat $T_{n}$ for all $n$, such that $P_{\mathcal{M}_{n}} \rightarrow P_{\mathcal{M}}$ in the strong operator topology. The concept needs little justification and has been examined by several previous authors. In the finite-dimensional setting it appears in [4] and [5]. Also, see Appendix 1 in [2] for a characterization of the stable invariant subspace of an operator on a finite-dimensional space. The idea of a stable invariant subspace is also implicit in [10] and [9]. In [1] and [3] a stronger concept of stable invariant

1991 Mathematics Subject Classification: 47A15, 47B15.

Key words and phrases: invariant subspace, stability, normal operators. 
subspace is considered, where, in the definition, it is required that the sequence of projections converges in norm rather than in the strong operator topology. Here we will call such spaces norm stable.

The concept of stability is also related to the examination of "Lat" as a function and its points of continuity. Because the underlying Hilbert space is separable, the strong operator topology is metrizable on bounded subsets of $\mathcal{B}(\mathcal{H})$. Thus $(\mathcal{P}, \mathrm{SOT})$ is a metric space. Let $\mathcal{C}$ denote the collection of closed subsets of $\mathcal{P}$ and furnish $\mathcal{C}$ with the Hausdorff metric defined by using the SOT metric. Thus we have a function Lat $: \mathcal{B}(\mathcal{H}) \rightarrow \mathcal{C}$. It is a good exercise in the definitions to show that this function is continuous at an operator $T$ if and only if every invariant subspace for $T$ is stable. For a finite-dimensional space, it was proved in [7] that Lat is continuous at $T$ if and only if $T$ is cyclic (or non-derogatory). Later in this paper it will be shown that Lat is continuous at a shift of finite multiplicity.

Similar comments apply if norm stable invariant subspaces are considered and, in the preceding discussion, the norm topology replaces the strong operator topology on the set of projections.

1. Preliminaries. Denote by $\operatorname{Lat}_{\mathrm{s}} T$ the collection of stable invariant subspaces of $T$ and by $\operatorname{Lat}_{\mathrm{ns}} T$ the norm stable invariant subspaces of $T$. Clearly, Lat Lis $T \subseteq \operatorname{Lat}_{\mathrm{s}} T$ and it is easy to check that Lat $\operatorname{Lin}_{\text {ns }} T$ contains both $\{0\}$ and $\mathcal{H}$. The proof of the first lemma is an exercise.

1.1. Lemma. For any operator $T, \operatorname{Lat}_{\mathrm{s}} T$ is strongly closed and $\operatorname{Lat}_{\mathrm{ns}} T$ is norm closed.

An obvious question is the following.

1.2. Question. For any operator $T$, is $\operatorname{Lat}_{\mathrm{s}} T$ the strong closure of Lat $_{\text {ns }} T$ ?

The results of this paper support an affirmative answer. It will be shown that this is the case for the unilateral shift of finite multiplicity and for normal operators. However, this does not constitute sufficient evidence to warrant a conjecture at this time. It is not known, for example, whether the answer to this question is affirmative for the unilateral shift of infinite multiplicity.

1.3. Lemma. If $T_{n} \rightarrow T(S O T), P_{n}=\operatorname{cl}\left(\operatorname{ran} T_{n}\right)$, and $P=\operatorname{cl}(\operatorname{ran} T)$, then $P_{n} P \rightarrow P(S O T)$.

Proof. For any vector $h$,

$$
\left\|P_{n} T h-T h\right\|=\left\|P_{n}\left(T h-T_{n} h\right)+T_{n} h-T h\right\| \leq 2\left\|T h-T_{n} h\right\| .
$$

Since $\left\|P_{n}\right\| \leq 1$ for all $n$, for any vector $f$ we have $\left\|P_{n} P f-P f\right\| \rightarrow 0$ as $n \rightarrow \infty$. 
1.4. Lemma. Let $\left\{E_{n}\right\}$ be a sequence of idempotents and assume that $\left\|E_{n}-E\right\| \rightarrow 0$. If $P_{n}$ is the orthogonal projection onto $\operatorname{ran} E_{n}$ and $P$ the orthogonal projection onto ran $E$, then $\left\|P_{n}-P\right\| \rightarrow 0$.

P r o of. We first establish the following.

ClAIM. If $E$ is an idempotent and $f$ is a continuous function on $[0, \infty)$ with $f(0)=0$ and $f(t)=1$ for $t \geq 1$, then $f\left(E E^{*}\right)$ is the projection onto ran $E$.

Indeed, if $\mathcal{M}=\operatorname{ran} E$ and we write $E$ as a $2 \times 2$ matrix with respect to the decomposition $\mathcal{H}=\mathcal{M} \oplus \mathcal{M}^{\perp}$, then

$$
E=\left(\begin{array}{cc}
1 & X \\
0 & 0
\end{array}\right) .
$$

Hence

The claim now easily follows.

$$
E E^{*}=\left(\begin{array}{cc}
1+X X^{*} & 0 \\
0 & 0
\end{array}\right) .
$$

If $\left\{E_{n}\right\}$ is a sequence of idempotents and $\left\|E_{n}-E\right\| \rightarrow 0$, then $\| E_{n} E_{n}^{*}-$ $E E^{*} \| \rightarrow 0$. If $f$ is a continuous function on $[0, \infty)$, it follows that $\| f\left(E_{n} E_{n}^{*}\right)-$ $f\left(E E^{*}\right) \| \rightarrow 0$. It is now apparent that the lemma follows from the claim.

When the spectrum of $T$ is not connected, $\operatorname{Lat}_{\mathrm{s}} T$ is non-trivial. To economize on words, we adopt the common practice of calling sets clopen if they are simultaneously closed and open.

1.5. Lemma. If $U$ is a relatively clopen subset of $\sigma(\mathrm{T})$, then the Riesz subspace of $T$ corresponding to $U$ belongs to Lat $_{n \mathrm{~s}} \mathrm{~T}$.

Proof. Assume that $\left\|T_{n}-T\right\| \rightarrow 0$. If $U$ and $V$ are open sets in the plane that both meet $\sigma(T)$ and satisfy $\operatorname{cl} U \cap \operatorname{cl} V=\emptyset$, then $U \cup V$ is an open set containing $\sigma(T)$. Thus for all sufficiently large $n, \sigma\left(T_{n}\right) \subseteq U \cup V$. Consequently, $U \cap \sigma\left(T_{n}\right)$ is a relatively clopen set. An examination of the definition of the Riesz idempotent of $T_{n}$ corresponding to $U$ shows that $E_{n}=E\left(T_{n} ; U\right) \rightarrow E(T ; U)=E$ in norm. An application of the preceding lemma shows that $E \mathcal{H} \in \operatorname{Lat}_{\text {ns }} T$.

The next lemma says that for surjective operators, the kernel is a norm stable invariant subspace.

1.6. Lemma. If $T$ is a surjective operator and $\left\|T_{n}-T\right\| \rightarrow 0$, then $\left\|\operatorname{ker} T_{n}-\operatorname{ker} T\right\| \rightarrow 0$.

Proof. Since $T$ is surjective, $T T^{*}$ is invertible; hence $T_{n} T_{n}^{*}$ is invertible for all sufficiently large $n$ and $\left(T_{n} T_{n}^{*}\right)^{-1} \rightarrow\left(T T^{*}\right)^{-1}$. Consider the polar decompositions $T=\left(T T^{*}\right)^{1 / 2} W$ and $T_{n}=\left(T_{n} T_{n}^{*}\right)^{1 / 2} W_{n}$. Thus $W_{n}=$ $\left(T_{n} T_{n}^{*}\right)^{-1 / 2} T_{n} \rightarrow W$. But $W_{n} W_{n}^{*}=\operatorname{ker} T_{n}$ and $W W^{*}=\operatorname{ker} T$. 
It is an elementary exercise that if $\left\{P_{n}\right\}$ is a sequence of projections and $\left\|P_{n}-P\right\| \rightarrow 0$, then for any projection $Q \leq P$, there are projections $Q_{n} \leq P_{n}$ such that $\left\|Q_{n}-Q\right\| \rightarrow 0$. With this the next corollary is immediate.

1.7. Corollary. If $T$ is surjective and $\mathcal{M} \leq \operatorname{ker} T$, then $\mathcal{M}$ is a norm stable invariant subspace for $T$.

The next result constitutes a small diversion, but one with some interest.

1.8. Proposition. If $\mathcal{B}(\mathcal{H})$ has the norm topology and $\mathcal{P}$ has the strong operator topology, then the following statements are equivalent:

(a) the map $X \rightarrow \operatorname{cl}(\operatorname{ran} X)$ is continuous at $T^{*}$;

(b) the map $X \rightarrow \operatorname{ker} X$ is continuous at $T$;

(c) $T$ is either surjective or injective.

Proof. (a) $\Rightarrow(\mathrm{c})$. Assume that (c) is false. We will construct a sequence of operators $\left\{X_{n}\right\}$ such that $\left\|X_{n}-T^{*}\right\| \rightarrow 0$ but $\left\{\operatorname{cl}\left(\operatorname{ran} X_{n}\right)\right\}$ does not converge to $\operatorname{cl}\left(\operatorname{ran} T^{*}\right)$ in the strong operator topology. To say that (c) is false is to say that $\left(\operatorname{ran} T^{*}\right)^{\perp}=\operatorname{ker} T \neq(0)$ and $\operatorname{ran} T \neq \mathcal{H}$.

First suppose that $\operatorname{ran} T$ is not dense and choose unit vectors $e$ in $\operatorname{ker} T$ and $f$ in $(\operatorname{ran} T)^{\perp}$. Define $X_{n}$ by $X_{n} h=T^{*} h+n^{-1}\langle h, f\rangle e$. Because $e \perp$ $\operatorname{ran} T^{*}, \operatorname{ran} X_{n}=\operatorname{ran} T^{*}+\mathbb{C} e$ and the sequence $\left\{X_{n}\right\}$ is as promised.

Now suppose that $\operatorname{ran} T$ is dense but not the whole space. Since $\operatorname{ran} T$ is not closed, neither is $\operatorname{ran} T^{*}$ ([6], VI.1.10). Hence 0 is in the left essential spectrum of $T^{*}$ and so for each integer $n \geq 1$ there is an infinite-dimensional space $\mathcal{M}_{n}$ such that $\sup \left\{\left\|T^{*} h\right\|: h \in \mathcal{M}_{n}\right.$ and $\left.\|h\|=1\right\}<1 / n$ ([6], XI.2.3). Let $S_{n}: \mathcal{M}_{n} \rightarrow \mathcal{M}_{n}$ be a backward unilateral shift of multiplicity 1 on $\mathcal{M}_{n}$ and let $e_{n}$ be a unit vector in $\operatorname{ker} S_{n}$. Fix a unit vector $e$ in $\operatorname{ker} T$ and define $X_{n}$ by

$$
X_{n} h= \begin{cases}T^{*} h & \text { when } h \in \mathcal{M}_{n}^{\perp}, \\ X_{n} h=T^{*} S_{n} h+n^{-1}\left\langle h, e_{n}\right\rangle e & \text { when } h \in \mathcal{M}_{n} .\end{cases}
$$

Thus $\left\|X_{n}-T^{*}\right\| \leq 3 n^{-1}$ and, since $S_{n}\left(\mathcal{M}_{n} \ominus e_{n}\right)=\mathcal{M}_{n}, \operatorname{cl}\left(\operatorname{ran} X_{n}\right)=$ $\operatorname{cl}\left(\operatorname{ran} T^{*}\right)+\mathbb{C} e$.

(c) $\Rightarrow$ (b). If we assume that $T$ is surjective, the result is in Lemma 1.6. If $T$ is injective, assume that $\left\{T_{n}\right\}$ is a sequence of operators such that $\left\|T_{n}-T\right\| \rightarrow 0$ and let $P_{n}=\operatorname{ker} T_{n}$. If $Q$ is any WOT cluster point of $\left\{P_{n}\right\}$, then $T_{n} P_{n} \rightarrow_{\mathrm{cl}} T Q$ (WOT), But $T_{n} P_{n}=0$ for each $n$, so $T Q=0$. Hence $Q=0$ and, as the unique WOT cluster point of $\left\{P_{n}\right\}, Q$ is the WOT limit of $\left\{P_{n}\right\}$. Hence we also have $P_{n} \rightarrow 0$ (SOT).

(b) $\Rightarrow(\mathrm{a})$. If $\left\|X_{n}-T^{*}\right\| \rightarrow 0$, then $\left\|X_{n}^{*}-T\right\| \rightarrow 0$ and so, by (b), $\operatorname{ker} X_{n}^{*} \rightarrow \operatorname{ker} T(\mathrm{SOT})$. Thus $\left(\operatorname{ker} X_{n}^{*}\right)^{\perp} \rightarrow(\operatorname{ker} T)^{\perp}(\mathrm{SOT})$, whence (a).

2. Shifts of finite multiplicity. In this section we will give a proof that for a shift $S$ of finite multiplicity, Lat $S=$ Lat $_{\mathrm{s}} S$. This was essentially 
obtained in [3], where the norm stable invariant subspaces of any shift $S$ are characterized. That paper shows that if $\mathcal{M}$ is a proper invariant subspace for $S^{*}$, with $S$ a shift of any multiplicity, then $\mathcal{M}$ is norm stable if and only if the spectral radius of $S^{*} \mid \mathcal{M}$, written $r\left(S^{*} \mid \mathcal{M}\right)$, is strictly less than 1 . If $\operatorname{dim} \mathcal{M}<\infty$, then this spectral radius must be less than 1 since $S^{*}$ has no eigenvalues on the unit circle. Conversely, if it is also assumed that $S$ has finite multiplicity, then the first lemma below shows that the only invariant subspaces of $S^{*}$ for which the corresponding spectral radius is less than 1 are the finite-dimensional ones. So it follows from [3] that the non-zero norm stable invariant subspaces for a shift of finite multiplicity are the invariant subspaces of finite codimension. As will be shown later in this section, for $S$ having finite multiplicity, the elements of Lat $S$ having finite codimension are strongly dense in Lat $S$; this shows that such a shift is a point of continuity of Lat.

It is not known whether this is true for a shift of infinite multiplicity. In particular, if $S$ is a shift of infinite multiplicity and $\mathcal{M} \in$ Lat $S^{*}$, it is unknown whether there is a sequence of subspaces $\left\{\mathcal{M}_{n}\right\}$ in Lat $S^{*}$ such that $r\left(S^{*} \mid \mathcal{M}_{n}\right)<1$ for all $n$ and $P_{\mathcal{M}_{n}} \rightarrow P_{\mathcal{M}}$ (SOT).

2.1. Lemma. If $S$ is a unilateral shift of finite multiplicity, $\mathcal{M} \in$ Lat $S$ such that $\mathcal{M}^{\perp}$ is infinite-dimensional, and $P=P_{\mathcal{M}}$, then $\left\|P^{\perp} S P^{\perp}\right\|=1=$ $r\left(P^{\perp} S P^{\perp}\right)$ (the spectral radius of $\left.P^{\perp} S P^{\perp}\right)$.

Proof. Let $T=P^{\perp} S P^{\perp}$; so $r(T) \leq\|T\| \leq 1$. Because $S$ has finite multiplicity, $\operatorname{dim} \operatorname{ker} S^{* n}<\infty$. Because $\mathcal{M}^{\perp}$ is infinite-dimensional, for each $n \geq 1$ there is a unit vector $g_{n}$ in $\mathcal{M}^{\perp}$ that is orthogonal to $\operatorname{ker} S^{* n}$. Hence $1=\left\|S^{* n} g_{n}\right\|=\left\|T^{* n} g_{n}\right\|$. So $\left\|T^{* n}\right\|=1$ and $\left\|T^{n}\right\|=1$. This implies that $r(T)=\lim _{n}\left\|T^{n}\right\|^{1 / n}=1$.

2.2. Proposition. If $T$ is any operator on the Hilbert space $\mathcal{H}$ with $\|T\|=1$ and $\mathcal{M}$ is an invariant subspace for $T$ such that there is a $\lambda$ in $\sigma(T \mid \mathcal{M}) \cap \sigma\left(P^{\perp} T \mid \mathcal{M}^{\perp}\right)$ with $|\lambda|=1$, then $\mathcal{M}$ is not norm stable.

Pro of. Without loss of generality we may assume that $\lambda=1$. If $\varepsilon, \delta>0$, the fact that 1 belongs to the approximate point spectrum of both $T \mid \mathcal{M}$ and $P^{\perp} T \mid \mathcal{M}^{\perp}$ implies there are unit vectors $f$ in $\mathcal{M}$ and $g$ in $\mathcal{M}^{\perp}$ such that $\|T f-f\|<\varepsilon$ and $\left\|P^{\perp} T g-g\right\|<\delta$. Now

$$
\begin{aligned}
\|P T g\|^{2} & =\|T g\|^{2}-\left\|P^{\perp} T g\right\|^{2} \leq 1-\left(\|g\|-\left\|P^{\perp} T g-g\right\|\right)^{2} \\
& <1-(1-\delta)^{2}=2 \delta-\delta^{2} .
\end{aligned}
$$

Thus $\|T g-g\|^{2}=\left\|P^{\perp} T g-g\right\|^{2}+\|P T g\|^{2}<2 \delta$. Take $\delta=\varepsilon^{2} / 2$ and let $e=(f+g) / \sqrt{2}$.

So for each $\varepsilon>0$ there is a unit vector $e_{\varepsilon}$ such that $\left\|T e_{\varepsilon}-e_{\varepsilon}\right\|<\varepsilon$, $\left\|e_{\varepsilon}-P e_{\varepsilon}\right\|=1 / \sqrt{2}$, and $\left\|P e_{\varepsilon}\right\|=1 / \sqrt{2}$. Put $\mathcal{L}_{\varepsilon}=\mathbb{C} e_{\varepsilon}$ and consider the 
representation of $T$ as a $2 \times 2$ matrix with respect to the decomposition $\mathcal{H}=\mathcal{L}_{\varepsilon} \oplus \mathcal{L}_{\varepsilon}^{\perp}$. It follows that there are numbers $\varepsilon_{1}$ and $\varepsilon_{2}$ that converge to 0 as $\varepsilon \rightarrow 0$ and operators $B_{\varepsilon}$ and $Z_{\varepsilon}$ with $\left\|B_{\varepsilon}\right\| \leq 1$ and $\left\|Z_{\varepsilon}\right\| \rightarrow 0$ as $\varepsilon \rightarrow 0$ such that

$$
T=\left(\begin{array}{cc}
1+\varepsilon_{1} & Z_{\varepsilon} \\
\varepsilon_{2} & B_{\varepsilon}
\end{array}\right)
$$

Let

$$
A_{\varepsilon}=\left(\begin{array}{cc}
1 & 0 \\
0 & (1-\varepsilon) B_{\varepsilon}
\end{array}\right) .
$$

It follows that $\left\|T-A_{\varepsilon}\right\| \rightarrow 0$ as $\varepsilon \rightarrow 0$. Since $\left\|(1-\varepsilon) B_{\varepsilon}\right\|<1$, Lat $A_{\varepsilon}=$ $\left\{(0), \mathcal{L}_{\varepsilon}\right\} \oplus$ Lat $B_{\varepsilon}$. If it were the case that $\mathcal{M}$ is norm stable, then there would be an $\mathcal{M}_{\varepsilon}$ in Lat $A_{\varepsilon}$ such that $\left\|P-P_{\varepsilon}\right\| \rightarrow 0$ as $\varepsilon \rightarrow 0$. But from the splitting property of Lat $A_{\varepsilon}$ we deduce that for every $\varepsilon$ either $\mathcal{L}_{\varepsilon} \leq \mathcal{M}_{\varepsilon}$ or $\mathcal{L}_{\varepsilon} \leq \mathcal{M}_{\varepsilon}^{\perp}$. Hence $\left\|P_{\varepsilon}-P\right\| \geq\left\|\left(P_{\varepsilon}-P\right) e_{\varepsilon}\right\| \geq 1 / \sqrt{2}$, a contradiction.

The next corollary is proved by combining the preceding proposition with Lemma 2.1.

2.3. Corollary. If $S$ is a shift of finite multiplicity and $\mathcal{M}$ is a norm stable invariant subspace, then $\operatorname{dim} \mathcal{M}^{\perp}<\infty$.

Let $H^{2}(\mathbb{D} ; \mathcal{H})$ denote the Hardy space of $\mathcal{H}$-valued analytic functions and let $H^{\infty}(\mathbb{D} ; \mathcal{B}(\mathcal{H}))$ be the space of bounded analytic $\mathcal{B}(\mathcal{H})$-valued functions. Say a function $Q$ in $H^{\infty}(\mathbb{D} ; \mathcal{B}(\mathcal{H}))$ is inner if $Q(z)$ is an isometry on $\mathcal{H}$ for a.e. $z$ in $\partial \mathbb{D}$. If $Q$ is an inner function in $H^{\infty}(\mathbb{D} ; \mathcal{B}(\mathcal{H}))$, let $E_{Q}$ be the orthogonal projection of $H^{2}(\mathbb{D} ; \mathcal{H})$ onto $Q H^{2}(\mathbb{D} ; \mathcal{H})$. It is a standard exercise that the linear span of functions of the form $z^{n} e$, where $n \geq 0$ and $e \in \mathcal{H}$, is dense in $H^{2}(\mathbb{D} ; \mathcal{H})$

2.4. Lemma. If $Q$ is an inner function in $H^{\infty}(\mathbb{D} ; \mathcal{B}(\mathcal{H}))$ and $E_{Q}$ is the orthogonal projection of $H^{2}(\mathbb{D} ; \mathcal{H})$ onto $Q H^{2}(\mathbb{D} ; \mathcal{H})$, then for any vector $e$ in $\mathcal{H}$ and any non-negative integer $n$,

$$
E_{Q}\left(z^{n} e\right)=Q \sum_{k=0}^{n} \frac{1}{k !} z^{n-k} Q^{(k)}(0)^{*} e .
$$

P r o of. If $h$ is the right hand side of the preceding equation, then, since $h$ is clearly in $Q H^{2}(\mathbb{D} ; \mathcal{H})$, it suffices to show that $z^{n} e-h \perp Q H^{2}(\mathbb{D} ; \mathcal{H})$. Thus the proof will be accomplished by showing that $\left\langle z^{n} e, z^{m} Q x\right\rangle=\left\langle h, z^{m} Q x\right\rangle$ for all $m \geq 0$ and all $x$ in $\mathcal{H}$. Now

$$
\left\langle z^{n} e, z^{m} Q x\right\rangle=\int z^{n-m}\langle e, Q(z) x\rangle d m(z) .
$$


If $f(z)=\langle Q(z) x, e\rangle$, then $f \in H^{2}$ and so

$$
\begin{aligned}
\int f(z) \bar{z}^{n-m} d m(z) & = \begin{cases}\frac{1}{(n-m) !} f^{(n-m)}(0) & \text { if } n \geq m, \\
0 & \text { if } n<m,\end{cases} \\
& = \begin{cases}\frac{1}{(m-n) !}\left\langle Q^{(n-m)}(0) x, e\right\rangle & \text { if } n \geq m, \\
0 & \text { if } n<m .\end{cases}
\end{aligned}
$$

Hence

$$
\left\langle z^{n} e, z^{m} Q x\right\rangle= \begin{cases}\frac{1}{(n-m) !}\left\langle e, Q^{(n-m)}(0) x\right\rangle & \text { if } n \geq m \\ 0 & \text { if } n<m\end{cases}
$$

Now

$$
\left\langle h, z^{m} Q x\right\rangle=\sum_{k=0}^{n} \frac{1}{k !} \int z^{n-k-m}\left\langle Q(z) Q^{(k)}(0)^{*} e, Q(z) x\right\rangle d m(z) .
$$

But $Q(z)$ is an isometry a.e. $[m]$, so

$$
\left\langle h, z^{m} Q x\right\rangle=\sum_{k=0}^{n} \frac{1}{k !} \int z^{n-k-m}\left\langle Q^{(k)}(0)^{*} e, x\right\rangle d m(z)=\left\langle z^{n} e, z^{m} Q x\right\rangle .
$$

2.5. Proposition. If $\operatorname{dim} \mathcal{H}<\infty$ and $Q, Q_{1}, Q_{2}, \ldots$ are inner functions in $H^{\infty}(\mathbb{D} ; \mathcal{B}(\mathcal{H}))$ and $Q_{m}(z) \rightarrow Q(z)$ in $\mathcal{B}(\mathcal{H})$ uniformly for $z$ in compact subsets of $\mathbb{D}$, then $E_{Q_{m}} \rightarrow E_{Q}(S O T)$ in $\mathcal{B}\left(H^{2}(\mathbb{D} ; \mathcal{H})\right)$.

Proof. Under the hypothesis, for every $k \geq 0, Q_{m}^{(k)}(0) \rightarrow Q^{(k)}(0)$ as $m \rightarrow \infty$. If $\left\{e_{1}, \ldots, e_{d}\right\}$ is a basis for $\mathcal{H}$, then $\left\{z^{n} e_{j}: n \geq 0,1 \leq j \leq d\right\}$ is a basis for $H^{2}(\mathbb{D} ; \mathcal{H})$. From the preceding lemma,

$$
\left\langle E_{Q_{m}}\left(z^{n} e_{j}\right), z^{p} e_{q}\right\rangle=\sum_{k=0}^{n} \frac{1}{k !} \int z^{n-k-p}\left\langle Q_{m}(z) Q^{(k)}(0)^{*} e_{j}, e_{q}\right\rangle d m(z) .
$$

It now follows that $\left\langle E_{Q_{m}}\left(z^{n} e_{j}\right), z^{p} e_{q}\right\rangle \rightarrow\left\langle E_{Q}\left(z^{n} e_{j}\right), z^{p} e_{q}\right\rangle$ for all possible $n, j, p$ and $q$. Since all the operators here are projections, $E_{Q_{m}} \rightarrow E_{Q}$ (SOT) in $\mathcal{B}\left(H^{2}(\mathbb{D} ; \mathcal{H})\right)$.

2.6. THEOREM. If $S$ is a unilateral shift of finite multiplicity, then every invariant subspace is stable.

P r o of. We begin by showing that the invariant subspaces of finite codimension are norm stable. To do this, it is somewhat more convenient to consider $T=S^{*}$ rather than $S$. So assume that $\mathcal{M}$ is a finite-dimensional invariant subspace of $T$. Thus $\sigma(T \mid \mathcal{M}) \subseteq \sigma_{p}(T) \subseteq \mathbb{D}$. Let $\sigma(T \mid \mathcal{M})=\left\{\lambda_{1}, \ldots, \lambda_{q}\right\}$, let $\mathcal{M}_{1}, \ldots, \mathcal{M}_{q}$ be the corresponding Riesz subspaces for $T \mid \mathcal{M}$, and let $k_{i}$ be the smallest positive integer such that $\left(T \mid \mathcal{M}_{i}-\lambda_{i}\right)^{k_{i}}=0$. Thus $\mathcal{M}_{i} \subseteq \operatorname{ker}\left(T-\lambda_{i}\right)^{k_{i}}$. 
Since $T$ is the adjoint of the shift and $\left|\lambda_{i}\right|<1,\left(T-\lambda_{i}\right)^{k}$ is surjective for every positive integer $k$. So if $\left\{T_{n}\right\}$ is a sequence of operators that converges to $T$, then

$$
\left\|\operatorname{ker}\left(T_{n}-\lambda_{i}\right)^{k_{i}}-\operatorname{ker}\left(T-\lambda_{i}\right)^{k_{i}}\right\| \rightarrow 0 .
$$

By Corollary 1.7, each $\mathcal{M}_{i}$ is norm stable. The fact that $\mathcal{M}_{i} \cap \mathcal{M}_{j}=(0)$ for $i \neq j$ and the linear span of $\mathcal{M}_{1}, \ldots, \mathcal{M}_{q}$ is the closed subspace $\mathcal{M}$ implies, by a small argument, that $\mathcal{M}$ is norm stable.

The proof will be completed by showing that these invariant subspaces are strongly dense in Lat $S$ and invoking Lemma 1.1. Represent $S$ as multiplication by the independent variable on $H^{2}(\mathbb{D} ; \mathcal{H})$, where $\mathcal{H}$ is a finitedimensional Hilbert space.

It is well known that the invariant subspaces for $S$ are precisely the subspaces of the form $Q H^{2}(\mathbb{D} ; \mathcal{H})$, for some $\mathcal{B}(\mathcal{H})$-valued inner function $Q$. By a result of Herrero [11], the fact that $\mathcal{H}$ is finite-dimensional implies that every inner function in $H^{\infty}(\mathbb{D} ; \mathcal{B}(\mathcal{H}))$ is the uniform limit of Blaschke products. But if $Q$ is a Blaschke product, then there is a sequence of Blaschke products such that $Q_{m}(z) \rightarrow Q(z)$ uniformly on compact subsets of $\mathbb{D}$ and each $Q_{m} H^{2}(\mathbb{D} ; \mathcal{H})$ has finite codimension in $H^{2}(\mathbb{D} ; \mathcal{H})$ (cf. [8]). The result now follows by the preceding proposition.

Note that the preceding theorem gives an affirmative answer to Question 1.2 when the operator is a unilateral shift of finite multiplicity. If $S$ is a unilateral shift of infinite multiplicity, the norm stable invariant subspaces of $S$ are characterized in [3], but it remains unknown whether Question 1.2 has an affirmative answer in this case.

3. Stable invariant subspaces for normal operators. Throughout this section $N$ will denote a normal operator with spectral decomposition $N=\int z d E(z)$. From $\S 1$ we know that Lat $N$ is strongly closed and contains the spectral subspaces corresponding to the clopen subsets of $\sigma(N)$. The main result of this section is that also the converse is true.

3.1. Theorem. For a normal operator $N$ on a separable Hilbert space $\mathcal{K}$, Lat $N$ consists of those spectral subspaces of $N$ that are strong limits of spaces of the form $E(U) \mathcal{H}$ for $U$ a clopen subset of $\sigma(N)$.

The norm stable invariant subspaces of a normal operator were characterized in [3] as the spectral subspaces corresponding to clopen subsets of $\sigma(N)$. This result is not needed here, nor are the results from the preceding section.

For any subset $X$ of the plane, let $(X)_{\varepsilon} \equiv\{z: \operatorname{dist}(z, X)<\varepsilon\}$.

3.2. LemmA. Let $N$ be a normal operator, let $\varepsilon>0$, and let $C_{1}, \ldots, C_{n}$ be components of $\sigma(N)$ such that $\sigma(N) \subseteq\left(C_{1}\right)_{\varepsilon} \cup \ldots \cup\left(C_{n}\right)_{\varepsilon}$. If $\left\{U_{1}, \ldots, U_{n}\right\}$ 
is a cover of $\sigma(N)$ by open sets with pairwise disjoint closures such that

(i) $U_{i} \cap \sigma(N)$ is clopen,

(ii) $C_{i} \subseteq U_{i} \subseteq \operatorname{cl} U_{i} \subseteq\left(C_{i}\right)_{\varepsilon}$,

(iii) $\sigma(N) \subseteq \bigcup_{i=1}^{n} U_{i}$, then there are operators $Q=\bigoplus_{i=1}^{n} Q_{i}$ and $R=$ $\bigoplus_{i=1}^{n} R_{i}$ on $\bigoplus_{i=1}^{n} E\left(U_{i}\right) \mathcal{H}$ that satisfy the following.

(a) $\|N-Q\|<2 \varepsilon$ and $\|N-R\|<2 \varepsilon$.

(b) For $1 \leq i \leq n, \sigma\left(Q_{i}\right)$ and $\sigma\left(R_{i}\right)$ are single points in $U_{i}$.

(c) For $1 \leq i \leq n$, Lat $Q_{i}=$ Lat $R_{i}^{*}$ and these lattices are nests.

Proof. Put $\mathcal{H}_{i}=E\left(U_{i}\right) \mathcal{H}$ and $N_{i}=N \mid \mathcal{H}_{i}$. There are two cases to consider depending on whether $\mathcal{H}_{i}$ is finite- or infinite-dimensional. If $\mathcal{H}_{i}$ is finite-dimensional, it suffices to consider the case where $U_{i}$ contains a single eigenvalue of $N$, say $\lambda$. So there is a basis for $\mathcal{H}_{i}$ with respect to which the matrix of $N_{i}$ is diagonal with entry $\lambda$. In this case we can take for $Q_{i}$ and $R_{i}$ the matrices

$$
Q_{i}=\left(\begin{array}{ccccc}
\lambda & \varepsilon & 0 & \ldots & 0 \\
0 & \lambda & \varepsilon & \ldots & 0 \\
\vdots & \ddots & \ddots & & \vdots \\
0 & 0 & \ldots & \lambda & \varepsilon \\
0 & 0 & \ldots & 0 & \lambda
\end{array}\right), \quad R_{i}=\left(\begin{array}{ccccc}
\lambda & 0 & 0 & \ldots & 0 \\
\varepsilon & \lambda & 0 & \ldots & 0 \\
\vdots & \ddots & \ddots & & \vdots \\
0 & \ldots & \varepsilon & \lambda & 0 \\
0 & \ldots & 0 & \varepsilon & \lambda
\end{array}\right) .
$$

Now assume that $\mathcal{H}_{i}$ is infinite-dimensional. Construct a smooth Jordan arc $\gamma_{i}:[0,1] \rightarrow U_{i}$ such that $C_{i} \subseteq\left(\gamma_{i}\right)_{\varepsilon}$.

Claim. There is a diagonal normal operator $M_{i}$ with $\sigma\left(M_{i}\right)=\gamma_{i}$ and $\left\|N_{i}-M_{i}\right\|<3 \varepsilon$.

Let $D_{i}$ be a diagonal normal operator with finite spectrum such that $\sigma\left(D_{i}\right) \subseteq \sigma\left(N_{i}\right)$, each eigenvalue of $D_{i}$ that belongs to $C_{i}$ has infinite multiplicity, $C_{i} \subseteq \sigma\left(D_{i}\right)_{\varepsilon}$, and $\left\|D_{i}-N_{i}\right\|<\varepsilon$. Let $\Gamma$ be a countable dense subset of $\gamma_{i}$. Thus each point of $\Gamma$ is within a distance $2 \varepsilon$ of infinitely many eigenvalues of $D_{i}$ (counting multiplicities) and every point of $\sigma\left(D_{i}\right)$ is within $2 \varepsilon$ of infinitely many points of $\Gamma$. Matching eigenvalues, we can find a diagonal normal operator $M_{i}$ such that $\left\|M_{i}-D_{i}\right\|<2 \varepsilon$ and whose eigenvalues are precisely the set $\Gamma$. This establishes the claim.

Let $A_{i}=\gamma_{i}^{-1}\left(M_{i}\right)$. So $A_{i}$ is a self-adjoint operator with spectrum $[0,1]$ and $\gamma_{i}\left(A_{i}\right)=M_{i}$. Let $p_{i}$ be a polynomial with $p_{i}^{\prime}(0) \neq 0$ such that $\mid p(t)-$ $\gamma_{i}(t) \mid<\varepsilon$ for all $t$ in $[0,1]$. Thus $\left\|p_{i}\left(A_{i}\right)-M_{i}\right\|<\varepsilon$. Let $B_{i}$ be a quasinilpotent operator with Lat $B_{i}$ totally ordered and such that $\left\|B_{i}-A_{i}\right\|$ and $\left\|B_{i}^{*}-A_{i}\right\|$ are sufficiently small that $\left\|p_{i}\left(B_{i}\right)-p_{i}\left(A_{i}\right)\right\|<\varepsilon$ and $\left\|p_{i}\left(B_{i}^{*}\right)-p_{i}\left(A_{i}\right)\right\|<\varepsilon$ (cf. [12]). Put $Q_{i}=p_{i}\left(B_{i}\right)$. Since $p_{i}^{\prime}(0) \neq 0$, it follows that $p_{i}$ is one-to-one near 0 and so $p_{i}^{-1}$ is a well-defined analytic function in a neighborhood of $\sigma\left(Q_{i}\right)=\left\{p_{i}(0)\right\}$. By Runge's Theorem, $B_{i}$ and $Q_{i}$ generate the same norm 
closed algebras, so that Lat $B_{i}=\operatorname{Lat} Q_{i}$. Similarly, if $R_{i}=p_{i}\left(B_{i}^{*}\right)$, then Lat $R_{i}=$ Lat $B_{i}^{*}=$ Lat $Q_{i}^{*}$ and $\left\|R_{i}-p_{i}\left(A_{i}\right)\right\|<\varepsilon$.

Letting $Q$ and $R$ be defined as in the statement of the lemma, we see that $\|N-Q\|<5 \varepsilon,\|N-R\|<5 \varepsilon$, and conditions (b) and (c) are satisfied.

3.3. Lemma. If $N$ is a normal operator and $\mathcal{M}$ is a stable invariant subspace, then $\mathcal{M}$ reduces $N$.

Proof. An application of the Spectral Theorem shows that there is a sequence $\left\{N_{k}\right\}$ of cyclic, reductive normal operators that converges to $N$ in norm. If $P$ is the projection onto $\mathcal{M}$, then there is a sequence of projections $\left\{P_{k}\right\}$ such that $P_{k} \in$ Lat $N_{k}$ and $P_{k} \rightarrow P$ (SOT). Since each $N_{k}$ is reductive, $N_{k} P_{k}-P_{k} N_{k}=0$. By taking limits we see that $N P=P N$ and so $\mathcal{M}$ reduces $N$.

Proof of Theorem 3.1. We already know from Lemma 1.5 that Lat $_{\mathrm{s}} N$ contains $E(U) \mathcal{H}$ for each clopen subset $U$ of $\sigma(N)$ and hence the strong limits of such spectral projections (Lemma 1.1). So let $\mathcal{M} \in \operatorname{Lat}_{\mathrm{s}} N$ and let $P$ be the projection of $\mathcal{H}$ onto $\mathcal{M}$.

For each positive integer $n$, let $\left\{C_{n i}: 1 \leq i \leq p_{n}\right\}$ be a collection of components of $\sigma(N)$ and let $\left\{U_{n i}: 1 \leq i \leq p_{n}\right\}$ be open sets such that the following are satisfied.

(i) $\operatorname{cl} U_{n i} \cap \operatorname{cl} U_{n j}=\emptyset$ for $i \neq j$.

(ii) $C_{n i} \subseteq U_{n i} \subseteq\left(C_{n i}\right)_{1 / n}$.

(iii) $\sigma(N) \subseteq \bigcup_{i=1}^{p_{n}} U_{n i}$.

Put $E_{n i}=E\left(U_{n i}\right)$ and $\mathcal{H}_{n i}=E_{n i} \mathcal{H}$. According to Lemma 3.2 we can find operators $Q_{n}=\bigoplus_{i=1}^{p_{n}} Q_{n i}$ and $R=\bigoplus_{i=1}^{p_{n}} R_{n i}$ on $\bigoplus_{i=1}^{p_{n}} \mathcal{H}_{n i}$ that satisfy the following.

(a) $\left\|N-Q_{n}\right\|<2 / n$ and $\left\|N-R_{n}\right\|<2 / n$.

(b) For $1 \leq i \leq p_{n}, \sigma\left(Q_{n i}\right)$ and $\sigma\left(R_{n i}\right)$ are single points in $U_{n i}$.

(c) Lat $Q_{n i}=$ Lat $R_{n i}^{*}$ for $1 \leq i \leq p_{n}$ and these lattices are nests.

From Lemma 3.3 we know that $P N=N P$ and so $P=\sum_{i} P E_{n i}=$ $\sum_{i} E_{n i} P$. Since $P$ is stable, there are projections $A_{n}$ and $B_{n}$ in Lat $Q_{n}$ and Lat $R_{n}$, respectively, such that $A_{n} \rightarrow P$ and $B_{n} \rightarrow P$ (SOT). From condition (b) we have $A_{n}=\bigoplus_{i=1}^{p_{n}} A_{n i}$ and $B_{n}=\bigoplus_{i=1}^{p_{n}} B_{n i}$ with $A_{n i}$ and $B_{n i}$ in Lat $Q_{n i}$ and Lat $R_{n i}$, respectively. Note that by (c), for each $i$ we see that either $A_{n i} \geq E_{n i}-B_{n i}$ or $A_{n i} \leq E_{n i}-B_{n i}$.

Let

$$
E_{n}=\sum\left\{E_{n i}: A_{n i} \geq E_{n i}-B_{n i}\right\} .
$$

By passing to a subsequence if necessary, we may assume that there is an operator $E$ with $0 \leq E \leq 1$ such that $E_{n} \rightarrow E$ in the weak operator topology 
(WOT). It will now be shown that $E=P$. Since each $E_{n}$ is a spectral projection this will imply that $E_{n} \rightarrow P$ (SOT), proving the theorem.

To do this, first observe that $E_{n} A_{n}=A_{n} E_{n} \geq E_{n}\left(1-B_{n}\right)=\left(1-B_{n}\right) E_{n}$. Now observe that for two commuting sequences of operators, one of which converges in the strong operator topology and the other in the weak operator topology, the product converges in the weak operator topology. Thus taking WOT limits in the above inequalities, we get $E P \geq E(1-P)$. Multiplying both sides by $1-P$ and again using commutativity, this implies that $0 \geq$ $(1-P) E$, which is a positive operator. Thus $E=E P=P E$. On the other hand,

$$
\left(1-E_{n}\right) A_{n}=A_{n} \sum\left\{E_{n i}: A_{n i} \leq E_{n i}-B_{n i}\right\} \leq E_{n}\left(1-B_{n}\right) .
$$

Again taking limits we get $(1-E) P \leq(1-E)(1-P)$. Multiplying both sides by $P$ we deduce that $(1-E) P=0$ or $P=P E$. Therefore $P=E$.

Recall from the introduction that an operator $T$ is a point of continuity of the function Lat $: \mathcal{H} \rightarrow \mathcal{C}$ if and only if Lat $T=\operatorname{Lat}_{\mathrm{s}} T$.

3.4. Corollary. A normal operator $N$ is a point of continuity of Lat if and only if $N$ is cyclic, reductive, and every spectral projection is the strong operator topology limit of a sequence of spectral projections corresponding to clopen subsets of $\sigma(N)$.

Proof. If $N=\int z d E(z)$ is a cyclic, reductive normal operator, then every invariant subspace is the range of a spectral projection. If it is also assumed that for each Borel set $\Delta$ there is a sequence $\left\{U_{n}\right\}$ of clopen subsets of $\sigma(N)$ such that $E\left(U_{n}\right) \rightarrow E(\Delta)$ (SOT), then Theorem 3.1 says that Lat $N=$ Lat $_{\mathrm{s}} N$.

Conversely, assume that $N$ is a point of continuity of Lat. It follows from Theorem 3.1 that each invariant subspace for $N$ reduces $N$ and that the projection onto this subspace is a spectral projection. Thus $N$ is cyclic and reductive. The rest of the corollary follows from the theorem.

Note that if a normal operator is a point of continuity of Lat, it is not necessary for it to have totally disconnected spectrum. For example, if $K$ is the compact set consisting of the closed unit interval together with the "snowflakes" $\{(1 / n, k / n): n \geq 1$ and $1 \leq k \leq n\}$ and $\mu$ is any measure assigning positive measure to each snowflake and no measure to the interval, then the normal operator $N=M_{z}$ on $L^{2}(\mu)$ is a point of continuity of Lat and $\sigma(N)=K$.

This paper concludes with a result that touches its subject matter but has an unusual hypothesis. Recall that an operator $T$ is biquasitriangular if for each scalar $\lambda$ such that $T-\lambda$ is semi-Fredholm, the Fredholm index of $T-\lambda$ is 0 . Theorem 6 of [9] shows that the closure of the set of unicellular 
operators (those for which the lattice of invariant subspaces is a nest) is the set of biquasitriangular operators with connected spectrum and essential spectrum. Thus for any biquasitriangular operator $T$ with connected spectrum and essential spectrum, Lat $T$ is linearly ordered. On the other hand, in [3] it was shown that for a biquasitriangular operator $T$ with connected spectrum and essential spectrum, Lat $_{n s} T$ is trivial.

This leads to the possibility that Question 1.2 has a negative answer for a biquasitriangular operator $T$ with connected spectrum and essential spectrum. The final result says that Question 1.2 is related with a somewhat better known problem in operator theory. Recall that an operator $T$ is transitive if it has no non-trivial invariant subspaces.

3.5. Proposition. If there exists a transitive operator and $T$ is a biquasitriangular operator with connected spectrum and essential spectrum, then Lat $_{\mathrm{s}} T$ is trivial.

Proof. It is shown in [9] that if there is a transitive operator, then the closure of the set of all transitive operators is the set of biquasitriangular operators with connected spectrum and essential spectrum. This proves the proposition.

Thus if the answer to Question 1.2 is negative for biquasitriangular operators with connected spectrum and essential spectrum, then every operator has a non-trivial invariant subspace. It would be interesting to know whether Question 1.2 is equivalent to the Invariant Subspace Problem.

\section{References}

[1] G. T. Adams, A nonlinear characterization of stable invariant subspaces, Integral Equations Operator Theory 6 (1983), 473-487.

[2] C. A postol, L. A. Fialkow, D. A. Herrero, and D. Voiculescu, Approximation of Hilbert Space Operators, Vol. II, Pitman Res. Notes Math. 102, Pitman, Boston, 1984.

[3] C. Apostol, C. Foiaş, and N. Salinas, On stable invariant subspaces, Integral Equations Operator Theory 8 (1985), 721-750.

[4] H. Bart, I. Gohberg, and M. A. Kaashoek, Stable factorizations of monic matrix polynomials and stable invariant subspaces, ibid. 1 (1978), 496-517.

[5] S. Campbell and J. Daughtry, The stable solutions of quadratic matrix equations, Proc. Amer. Math. Soc. 74 (1979), 19-23.

[6] J. B. Conway, A Course in Functional Analysis, Springer, New York, 1990.

[7] J. B. Conway and P. R. Halmos, Finite-dimensional points of continuity of Lat, Linear Algebra Appl. 31 (1980), 93-102.

[8] Yu. P. Ginzburg, The factorization of analytic matrix functions, Dokl. Akad. Nauk SSSR 159 (3) (1964), 489-492 (in Russian).

[9] D. W. Hadwin, An addendum to limsups of lats, Indiana Univ. Math. J. 29 (1980), 313-319. 
[10] P. R. Halmos, Limsups of lats, ibid., 293-311.

[11] D. A. Herrero, Inner functions under uniform topology, II, Rev. Un. Mat. Argentina 28 (1976), 23-35.

[12] -, Approximation of Hilbert Space Operators, I, Pitman, London, 1982.

Mathematics

University of Tennessee

Knoxville, Tennessee 37996-1300

U.S.A.

E-mail: conway@novell.math.utk.edu

Web: http://www.math.utk.edu/ ${ }^{\text {conway/ }}$
Department of Mathematics

University of New Hampshire Durham, New Hampshire 03824

U.S.A.

E-mail:don@christa.unk.edu 